T. Hibi

Nagoya Math. J.

Vol. 107 (1987), 91-119

\title{
UNION AND GLUEING OF A FAMILY OF COHEN-MACAULAY PARTIALLY ORDERED SETS
}

\author{
TAKAYUKI HIBI
}

Summary. By means of simple exact sequences in commutative algebra, we can derive some effective criteria for CohenMacaulay property of finite partially ordered sets.

\section{Introduction}

Given a finite partially ordered set (poset for short), for example,

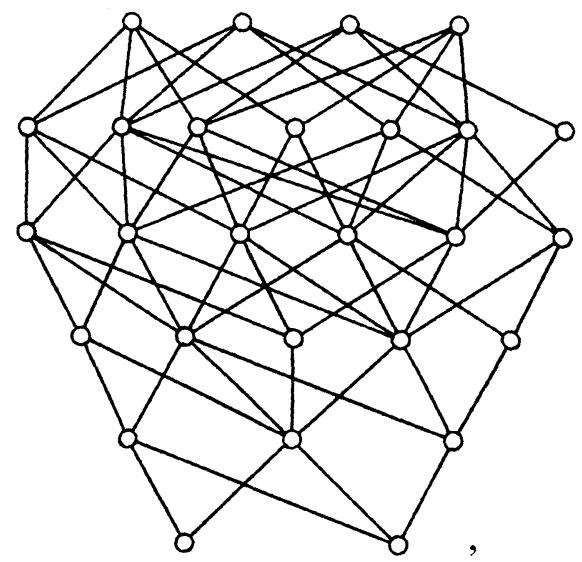

Figure 1

are there any effective criteria for Cohen-Macaulayness of it? This question is our main motivation to organize this paper. Why is this question so important? Because we have a conjecture that every "integral lattice" (or poset) is Cohen-Macaulay, which is proposed in [15, $\S 2, d$ )]. This conjecture is quite open except one partial affirmative answer obtained in [17, § 2, Corollary].

Historically, the notion of Cohen-Macaulay posets originated in

Received April 7, 1986. 
Baclawski [1] and [2]. Baclawski's definition is purely topological and is influenced by Folkman's result [11, Theorem 4.1], which in current terminology says that a geometric lattice is Cohen-Macaulay. On the other hand, Stanley [26] and Reisner [24] independently gave the ringtheoretical definition of Cohen-Macaulay posets (or complexes) under an influence of a commemorable work of Hochster [19].

The foundation in a theory of Cohen-Macaulay posets is a pioneering work of Reisner [24], in which it is proved that one can define CohenMacaulay posets by using either topology or ring theory. Also, the proof by Stanley [26] of the upper bound conjecture for spheres is one of the most dramatic applications of commutative algebra to combinatorics.

In this paper, we will apply local cohomology theory in commutative algebra to a theory of Cohen-Macaulay posets and complexes. Since our tool is based on only depth sensitivity and long exact sequences of local cohomology modules, the method is quite simple and rather formal.

This paper is divided into four sections. In Section 1, after recalling some basic definitions and terminology from commutative algebra and combinatorics, we shall analyze two exact sequences concerning with Stanley-Reisner rings of simplicial complexes, which will play essential roles throughout all of this paper.

In Section 2, based on the exact sequences considered in Section 1, from a purely ring-theoretical viewpoint, we shall give quite simple and elementary alternative proofs to well-known and important combinatorial results, the rank selection theorem and Cohen-Macaulayness of shellable complexes and $G$-complexes.

On the other hand, Section 3 is a purely combinatorial content, which is also associated with graph theory. In order to apply the argument for simplicial complexes, contained in Section 1, to finite partially ordered sets in Section 4, we shall define explicitly an "intersection", a "union" and the "glueing" of a family of partially ordered sets.

Finally, in Section 4, we shall state our criteria for Cohen-Macaulay property of finite partially ordered sets and, as an example of applications, we shall treat the partially ordered set $\Pi_{n}$ of partitions of the integer $n$ ordered by refinement. Also, we shall consider a question that what conditions of partially ordered sets ensure Buchsbaum posets to be Cohen-Macaulay.

It seems likely impossible that we can apply our criteria to all finite 
partially ordered sets. Nevertheless, we hope that our criteria will turn out to be useful in a theory of Cohen-Macaulay posets in our further work, see [34].

\section{§1. $0 \rightarrow \oplus_{i=1}^{n} k\left[\operatorname{star}_{\Delta}\left(\sigma_{i}\right)\right] \rightarrow k[\Delta] \rightarrow k\left[\Delta^{\prime}\right] \rightarrow 0$}

In this section we shall consider mainly an exact sequence stated in the above title.

(1.1) To begin with, we will recall some fundamental definitions and results on Stanley-Reisner rings $k[\Delta]$.

Let $V$ be a finite set, called the vertex set, and $\Delta$ a simplicial complex on $V$. Thus $\Delta$ is a family of subsets of $V$ satisfying (i) $\{v\} \in V$ for all $v \in V$ and (ii) $\sigma \in \Delta, \tau \subset \sigma$ imply $\tau \in \Delta$.

Let $k$ be a field and $A=k[v ; v \in V]$ the polynomial ring in $\sharp(V)$ variables over $k$, where $\sharp(V)$ means the cardinality of $V$ as a set. By abuse of notation, we are regarding the vertices $v$ as indeterminates over $k$. Define $I_{\Delta}$ to be the ideal of $A$ generated by all square-free monomials $v_{i_{1}} v_{i_{2}} \cdots v_{i_{r}}$ such that $\left\{v_{i_{1}}, v_{i_{2}}, \cdots, v_{i_{r}}\right\} \notin \Delta$, and $k[\Delta]:=A / I_{\Delta}$. The $k$-algebra $k[\Delta]$ is called the Stanley-Reisner ring on $\Delta$ in commemoration of Stanley [26] and Reisner [24]. From now on, we will consider $A$ and $k[\Delta]$ as graded rings over $k$ with the standard grading, i.e., the degree of each $v \in V$ is one.

An element $\sigma \in \Delta$ is called a face and its dimension $\operatorname{dim} \sigma$ is $\sharp(\sigma)$. A maximal face, with respect to inclusion, is also called a facet. The dimension of $\Delta$, denoted by $\operatorname{dim} \Delta$, is $\max \{\operatorname{dim} \sigma ; \sigma \in \Delta\}$. Note that $\operatorname{dim} \sigma$ and $\operatorname{dim} \Delta$ in this paper are one more than those in [2] or [10]. Also, $\Delta$ is called pure if $\operatorname{dim} \sigma=\operatorname{dim} \Delta$ for all facets $\sigma \in \Delta$.

We will denote by $\operatorname{dim} k[\Delta]$ (resp. depth $k[\Delta]$ ) the dimension (resp. depth) of $k[4]$ as a graded $k$-algebra. It can be checked, see Stanley [30, p. 63], that $\operatorname{dim} k[\Delta]$ coincides with $\operatorname{dim} \Delta$. In the following, we often regard $k[\Delta]$ as a graded $A$-module, in this case, of course, $\operatorname{dim} k[\Delta]$ (resp. depth $k[\Delta]$ ) coincides with $\operatorname{dim}_{A} k[\Delta]$ (resp. depth $A$ [ $[\Delta]$ ), the dimension (resp. depth) of $k[\Delta]$ as a graded $A$-module. Note that depth $k[\Delta]$ is always positive since $k[\Delta]$ is a reduced $k$-algebra.

A simplicial complex $\Delta$ is called Cohen-Macaulay over a field $k$ if $k[\Delta]$ is a Cohen-Macaulay ring. Express the ideal $I_{\Delta}$ as an intersection of prime ideals in $A$, and we see that every Cohen-Macaulay complex $\Delta$ is pure, see Reisner [24, Lemma 9]. 
(1.2) Let $\Delta$ be a simplicial complex on the vertex set $V$. For $\sigma \in \Delta$, define

$$
\begin{aligned}
& \operatorname{link}_{\Delta}(\sigma)=\{\tau \in \Delta ; \sigma \cap \tau=\varnothing, \sigma \cup \tau \in \Delta\} \\
& \operatorname{star}_{\Delta}(\sigma)=\{\tau \in \Delta ; \sigma \cup \tau \in \Delta\}
\end{aligned}
$$

Then we have

$$
k\left[\operatorname{star}_{\Delta}(\sigma)\right]=k\left[\operatorname{link}_{\Delta}(\sigma)\right][v ; v \in \sigma] .
$$

By Hochster [20, (5.6)], both $\operatorname{link}_{4}(\sigma)$ and $\operatorname{star}_{4}(\sigma)$ are Cohen-Macaulay if $\Delta$ is Cohen-Macaulay.

Now consider the following graded $A$-module homomorphism $\Phi_{\sigma}$ defined by

$$
\begin{gathered}
\Phi_{\sigma}: A(-\#(\sigma)) \stackrel{\sigma}{\longrightarrow} k[\Delta] \\
\qquad \longmapsto \\
f \longmapsto
\end{gathered}
$$

where $\sigma \in \Delta$. Refer to Goto-Watanabe [14, P. 181] for the definition of $A(-\#(\sigma))$. Since there is no confusion, for simplicity, we will write $A$ instead of $A(-\#(\sigma))$. What is the kernel of $\Phi_{\sigma}$ ? Let $f=\prod_{i} v_{i}^{n_{i}}$ be a monomial in $A$, then $\Phi_{\sigma}(f)=0$ if and only if $\operatorname{Supp}(f):=\left\{v_{i} ; n_{i}>0\right\} \notin \operatorname{star}_{\Delta}(\sigma)$. Hence we obtain an injection

$$
\Phi_{\sigma}: k\left[\operatorname{star}_{\Delta}(\sigma)\right] \stackrel{\sigma}{\longrightarrow} k[\Delta]
$$

as graded $A$-modules.

Moreover, if $\sigma_{1}, \sigma_{2}, \cdots, \sigma_{n} \in \Delta$ then we have

$$
\begin{aligned}
\Phi_{\left(\sigma_{1}, \sigma_{2}, \cdots, \sigma_{n}\right)}: \bigoplus_{i=1}^{n} k\left[\operatorname{star}_{\Delta}\left(\sigma_{i}\right)\right] \stackrel{\left(\sigma_{1}, \sigma_{2}, \cdots, \sigma_{n}\right)}{\longrightarrow} k[\Delta] \\
w \\
\left(f_{1}, f_{2}, \cdots, f_{n}\right) \longmapsto \sum_{i=1}^{n}\left\{f_{i} \prod_{v \in \sigma_{i}} v\right\} .
\end{aligned}
$$

However, this map $\Phi_{\left(\sigma_{1}, \sigma_{2}, \cdots, \sigma_{n}\right)}$ is not necessarily injective and it is easy to see that

Lemma. $\Phi_{\left(\sigma_{1}, \sigma_{2}, \cdots, \sigma_{n}\right)}$ is injective if and only if $\sigma_{i} \cup \sigma_{j} \notin \Delta$ for all $i \neq j$.

What is the cokernel of $\Phi_{\left(\sigma_{1}, \sigma_{2}, \cdots, \sigma_{n}\right)}$ ? The image of $\Phi_{\left(\sigma_{1}, \sigma_{2}, \cdots, \sigma_{n}\right)}$ is an ideal of $k[\Delta]$ which is generated by $\sigma_{1}, \sigma_{2}, \cdots, \sigma_{n}$. Hence the cokernel of $\Phi_{\left(\sigma_{1}, \sigma_{2}, \cdots, \sigma_{n}\right)}$ is 


$$
k\left[\Delta-\left\{\tau \in \Delta ; \tau \supset \sigma_{i} \text { for some } i\right\}\right] .
$$

Summarizing the above observations, we have

Theorem. Let $\Delta$ be a simplicial complex on the vertex set $V$ and $\sigma_{1}, \sigma_{2}, \cdots, \sigma_{n}$ faces of $\Delta$ satisfying $\sigma_{i} \cup \sigma_{\jmath} \notin \Delta$ for all $i \neq j$. Then we have the exact sequence

$$
0 \longrightarrow \bigoplus_{i=1}^{n} k\left[\operatorname{star}_{d}\left(\sigma_{i}\right)\right] \longrightarrow k[\Delta] \longrightarrow k\left[\Delta^{\prime}\right] \longrightarrow 0
$$

as graded A-modules, where $A=k[v ; v \in V]$ and

$$
\Delta^{\prime}=\Delta-\left\{\tau \in \Delta ; \tau \supset \sigma_{i} \text { for some } i\right\} .
$$

(1.3) Let $A=\oplus_{n \geq 0} A_{n}$ be a noetherian graded ring defined over a field $k=A_{0}, m=A_{+}=\oplus_{n>0} A_{n}$, and $M=\oplus_{n \in Z} M_{n}$ a finitely generated graded $A$-module. We denote by $\underline{H}_{m}^{i}(M)$ the $i$-th local cohomology module, that is to say,

$$
\underline{H}_{m}^{i}(M)=\underline{\lim }_{\nu} \operatorname{Ext}_{A}^{i}\left(A / m^{\nu}, M\right),
$$

see Goto-Watanabe [14, P. 187].

Recall some fundamental properties of $\underline{H}_{m}^{i}(M)$. First, if $d=\operatorname{dim}_{A} M$ and $t=\operatorname{depth}_{A} M$, then $\underline{H}_{m}^{i}(M)=0$ unless $t \leq i \leq d$ and $\underline{H}_{m}^{d}(M) \neq 0$ and $\underline{H}_{m}^{t}(M) \neq 0$. Secondly, if

$$
0 \longrightarrow L \longrightarrow M \longrightarrow N \longrightarrow 0
$$

is an exact sequence of graded $A$-modules, then there exists a long exact sequence

$$
\begin{aligned}
& 0 \longrightarrow \underline{H}_{m}^{0}(L) \longrightarrow \underline{H}_{m}^{0}(M) \longrightarrow \underline{H}_{m}^{0}(N) \\
& \longrightarrow \underline{H}_{m}^{1}(L) \longrightarrow \underline{H}_{m}^{1}(M) \longrightarrow \underline{H}_{m}^{1}(N) \\
& \longrightarrow \cdots \\
& \longrightarrow \underline{H}_{m}^{i}(L) \longrightarrow \underline{H}_{m}^{i}(M) \longrightarrow \underline{H}_{m}^{i}(N) \\
& \longrightarrow \ldots
\end{aligned}
$$

of local cohomology modules.

Combining the above local cohomology theory with Theorem (1.2), we obtain the following

Corollary. Let $\Delta$ be a pure simplicial complex of dimension $d$ and $\sigma_{1}, \sigma_{2}, \cdots, \sigma_{n}$ faces of $\Delta$ satisfying $\sigma_{i} \cup \sigma_{j} \notin \Delta$ for all $i \neq i$. Also, let $\Delta^{\prime}=$ $\Delta-\left\{\tau \in \Delta ; \tau \supset \sigma_{i}\right.$ for some $\left.i\right\}$. 
a) If $\Delta$ is Cohen-Macaulay and $\operatorname{dim} \Delta^{\prime}<d$, then $\operatorname{dim} \Delta^{\prime}=d-1$ and $\Delta^{\prime}$ is also Cohen-Macaulay.

b) If $\operatorname{star}_{\Delta}\left(\sigma_{i}\right)$ are Cohen-Macaulay for all $i$ and $\Delta^{\prime}$ is Cohen-Macaulay of dimension $d$, then $\Delta$ is also Cohen-Macaulay.

Next, we will state two basic results on Buchsbaum rings and modules from Stückrad-Vogel [31] and Schenzel [25].

Lemma A (Stückrad-Vogel). Let $A=\oplus_{n \geq 0} A_{n}$ be a noetherian graded ring defined over a field $k=A_{0}, \quad m=\oplus_{n>0} A_{n}$ and $M=\oplus_{n \in Z} M_{n} \quad a$ finitely generated graded A-module of dimension $d$. Then $M$ is a Buchsbaum A-module if the natural map

$$
\underline{\operatorname{Ext}}_{A}^{i}(A / m, M) \longrightarrow \underline{H}_{m}^{i}(M)
$$

is surjective for all $i \neq d$. Moreover, if $A$ is a polynomial ring over $k$ then the converse is also true.

The above lemma is called the surjectivity criterion for Buchsbaum modules.

A simplicial complex $\Delta$ is called Buchsbaum over $k$ if $k[\Delta]$ is a Buchsbaum ring, which is equivalent to the fact that $k[\Delta]$ is a Buchsbaum $A$-module, where $A=k[v ; v \in V]$ as usual.

Lemma B (Schenzel). A simplicial complex $\Delta$ is Buchsbaum if and only if, for every $\sigma \in \Delta$, except $\sigma=\varnothing, \operatorname{link}_{4}(\sigma)$ is Cohen-Macaulay.

A Buchsbaum complex $\Delta$ is also called almost Cohen-Macaulay in Baclawski [2].

(1.4) Thanks to the exact sequence (*) of (1.2) and Corollary (1.3), we can give quite simple ring-theoretical proofs to important combinatorial results, which we shall achieve in Section 2. Here we treat only two extreme cases.

Example A. Let $\Delta$ be a pure simplicial complex of dimension $d$, $\sigma_{1}, \sigma_{2}, \cdots, \sigma_{n}$ the set of all facets (maximal faces) of $\Delta$ and

$$
\Delta_{d-1}=\{\tau \in \Delta ; \#(\tau) \leq d-1\} .
$$

In this case, the exact sequence $(*)$ is nothing but

$$
0 \longrightarrow \oplus_{i=1}^{n} k\left[v ; v \in \sigma_{i}\right] \longrightarrow k[\Delta] \longrightarrow k\left[\Delta_{d-1}\right] \longrightarrow 0
$$


which is considered in Baclawski-Garsia [4, P. 178]. Since $\operatorname{dim} k\left[\Delta_{d-1}\right]=$ $d-1$, by Corollary (1.3) a), $k\left[\Delta_{d-1}\right]$ is Cohen-Macaulay if so is $\Delta$.

On the other hand, suppose that $\Delta$ is Buchsbaum. We have the following commutative diagram

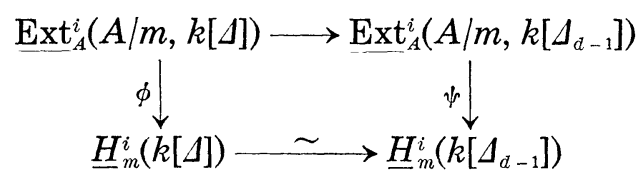

with natural maps if $i<d-1$, where $m$ is the irrelevant ideal of the polynomial ring $A$ over $k$ whose indeterminates are the vertices of $A$. Since $\phi$ is surjective by Lemma A of (1.3), $\psi$ must be surjective, hence $k\left[\Delta_{d-1}\right]$ is also Buchsbaum again by Lemma $\mathrm{A}$ of (1.3).

Repeating the above procedure, we see that if $\Delta$ is Cohen-Macaulay (resp. Buchsbaum) of dimension $d$, then

$$
\Delta_{i}=\{\tau \in \Delta ; \#(\tau) \leq i\}
$$

is also Cohen-Macaulay (resp. Buchsbaum) for all $i<d$, see BaclawskiGarsia [4, Theorem 5.5].

Example B. Let $\Delta$ be a pure simplicial complex on the vertex set $V$ and $v \in V$. Then, by $(*)$ we have

$$
0 \longrightarrow k\left[\operatorname{star}_{\lrcorner}(\{v\})\right] \longrightarrow k[\Delta] \longrightarrow k[\Delta] /(v) \longrightarrow 0 .
$$

Hence if both the localization $k[U]_{v}\left(=k\left[\operatorname{link}_{4}(\{v\})\right]\left[v, v^{-1}\right]\right)$ and the quotient $k[\Delta] /(v)$ are Cohen-Macaulay, then $k[\Delta]$ is also Cohen-Macaulay by Corollary (1.3) b). Note that if $\operatorname{dim}(k[\Delta] /(v))<\operatorname{dim} k[\Delta]$ then $\Delta=\operatorname{star}_{\alpha}(\{v\})$.

This result is false in general. For example, let $A=k[x, y] /\left(x y, y^{2}\right)$, $v=x \quad$ (resp. $A=k[x, y, z] /(x z, y z), v=x+y)$, then $A$ is not CohenMacaulay, however, both $A_{v}=k\left[x, x^{-1}\right]$ (resp. $k[x, y, 1 /(x+y)]$ ) and $A /(v)$ $=k[y] /\left(y^{2}\right)($ resp. $k[x, z] /(x z))$ are Cohen-Macaulay.

(1.5) Let $V$ be a finite set which is a union (not necessarily a disjoint union) $\bigcup_{i=1}^{n} V_{i}$ of non-empty subsets $V_{i}$ of $V$ and $\Delta_{i}$ simplicial complexes on the vertex sets $V_{i}(1 \leq i \leq n)$. Define $\Delta=\bigcup_{i=1}^{n} \Delta_{i}$, which is a simplicial complex on the vertex set $V$. Note that $\operatorname{dim} \Delta=\max \left\{\operatorname{dim} \Delta_{i} ; 1 \leqq i \leqq n\right\}$ and that $\Delta$ is pure if each $\Delta_{i}$ is pure with $\operatorname{dim} \Delta_{i}=\operatorname{dim} \Delta$.

When $\Delta=\bigcup_{i=1}^{n} \Delta_{i}$ turns out to be Cohen-Macaulay? For simplicity, we treat the case of $n=2$. 
Lemma. Let $n=2$. We have the natural exact sequence

$$
0 \longrightarrow k\left[\Delta_{1} \cup \Delta_{2}\right] \longrightarrow k\left[\Delta_{1}\right] \oplus k\left[\Delta_{2}\right] \longrightarrow k\left[\Delta_{1} \cap \Delta_{2}\right] \longrightarrow 0
$$

of graded modules over $A=k[v ; v \in V]$.

The above lemma was first considered in Hochster [19].

Again, combining the above exact sequence (**) with the local cohomology theory of (1.3), we immediately have the following

Corollary. Let $\Delta_{1}, \Delta_{2}$ be Cohen-Macaulay complexes of dimension $d$. Then $\Delta_{1} \cup \Delta_{2}$ is Cohen-Macaulay if and only if $\operatorname{depth} k\left[\Delta_{1} \cap \Delta_{2}\right] \geq d-1$.

ExAmple A. Let $\Delta$ be a simplicial complex on the vertex set $V$ and $\sharp(V) \geq 2$. Then depth $k[\Delta] \geq 2$ if and only if the geometric realization $|\Delta|$ is connected.

To see why this is true, first assume that $|\Delta|$ is not connected. Then, there exist non-empty subsets $V_{1}$ and $V_{2}$ of $V$ and simplicial complexes $\Delta_{i}$ on $V_{i}(i=1,2)$ such that $V=V_{1} \cup V_{2}, V_{1} \cap V_{2}=\varnothing$ and $\Delta=\Delta_{1} \cup \Delta_{2}$. Hence by $(* *)$ we have the exact sequence

$$
0 \longrightarrow k[\Delta] \longrightarrow k\left[\Delta_{1}\right] \oplus k\left[\Delta_{2}\right] \longrightarrow k \longrightarrow 0,
$$

so $\underline{H}_{m}^{1}(k[\Delta]) \neq 0$.

Conversely, suppose that $|\Delta|$ is connected. We shall prove depth $k[\Delta]$ $\geq 2$ by induction on $\sharp(V)$. We can choose $v \in V$ such that the geometric realization of the subcomplex $\Delta^{\prime}=\{\tau \in \Delta ; v \notin \tau\}$ on the vertex set $V-\{v\}$ is connected. By assumption of induction $\operatorname{depth}\left(k\left[\operatorname{star}_{4}(\{v\})\right]\right) \geq 2$ and depth $k\left[\Delta^{\prime}\right] \geq 2$, hence $\underline{H}_{m}^{i}(k[\Delta])=0$ if $i<2$, see the exact sequence contained in Example B of (1.4).

ExAmple B. Let $\Delta$ be a simplicial complex on the vertex set $V$. The extension of $\Delta$ by an element $v \in V$, denoted by $\Delta \propto v$, is a simplicial complex on $V \cup\left\{v^{\prime}\right\}\left(v^{\prime} \notin V\right)$ such that (i) $\sigma^{\prime}=\left\{v^{\prime}, v_{1}, \cdots, v_{i}\right\} \in \Delta \propto v$ if and only if $\sigma=\left\{v, v_{1}, \cdots, v_{i}\right\} \in \Delta$, where $v_{1}, \cdots, v_{i} \in V-\{v\}$, (ii) for $\sigma \subset V-\{v\}$, $\sigma \in \Delta \propto v$ if and only if $\sigma \in \Delta$, and (iii) $\left\{v, v^{\prime}\right\} \notin \Delta \propto v$. If $\Delta$ is Cohen-Macaulay, then so is $\Delta \propto v$.

To see this, let $\Delta^{\prime}=\{\tau \in \Delta \propto v ; v \notin \tau\}$. Then $\Delta \propto v=\Delta \cup \Delta^{\prime}$. Hence, thanks to the above corollary, we have only to check that depth $k\left[\Delta \cap \Delta^{\prime}\right]$ $\geq \operatorname{dim} k[\Delta]-1$. This inequality follows by the exact sequence contained in Example B of (1.4), since $k\left[\Delta \cap \Delta^{\prime}\right]=k[\Delta] /(v)$. 


\section{§2. Rank selection theorem, shellable complexes and $G$-complexes}

Based on the exact sequences $\left(^{*}\right)$ and $\left(^{* *}\right)$ of Section 1 , in this section, we shall give quite elementary proofs to some combinatorial results on Cohen-Macaulay posets and complexes.

(2.1) We begin with some definitions and terminology on partially ordered sets.

All posets to be considered are finite. The length of a chain (totally ordered set) $C$ is the cardinality $\sharp(C)$ as a set. The rank of a poset $Q$, denoted by rank $(Q)$, is the supremum of lengths of chains contained in $Q$. The rank of an element $\alpha$ in a poset is the supremum of lengths of chains descending from $\alpha$, and written by $r(\alpha)$. If two elements $\alpha$ and $\beta$ in a poset are incomparable, then we will write $\alpha \neq \beta$. A clutter is a poset in which no two elements are comparable. A poset ideal in a poset $Q$ is a subset $I$ such that $\alpha \in I, \beta \in Q$ and $\beta \leq \alpha$ together imply $\beta \in I$. A poset $P$ is called a subposet of $Q$ if $P \subset Q$ and, for $\alpha, \beta \in P, \alpha<\beta$ in $P$ if and only if $\alpha<\beta$ in $Q$.

When we regard a poset $Q$ only as a finite set $V$ forgetting the partial order, we call $V$ the underlying set of $Q$ and $Q$ is called a poset on $V$.

Let $Q$ be a poset on $V$ and define $\Delta(Q)$, called the order complex of $Q$, to be the simplicial complex on $V$ whose faces are the chains of $Q$. We will use such terminology as " $Q$ is pure" or " $Q$ is Cohen-Macaulay" to mean the corresponding statement for $\Delta(Q)$. Thus $Q$ is pure if and only if all maximal chains of $Q$ have the same length. Note that $\operatorname{rank}(Q)$ $=\operatorname{dim} \Delta(Q)$. Moreover, a Cohen-Macaulay (resp. Buchsbaum) poset $Q$ is one for which $\Delta(Q)$ is a Cohen-Macaulay (resp. Buchsbaum) complex. The Stanley-Reisner ring of a poset $Q$, denoted by $k[Q]$, is the StanleyReisner ring $k[\Delta(Q)]$ of $\Delta(Q)$, thus

$$
k[Q]=k[\alpha ; \alpha \in Q] /(\alpha \beta ; \alpha \nsim \beta) .
$$

Also, as an analogue of simplicial complexes, if $C$ is a chain of a poset $Q$ then we define

$$
\begin{aligned}
& \operatorname{link}_{Q}(C)=\{\alpha \in Q ; \alpha \notin C \text { and } C \cup\{\alpha\} \in \Delta(Q)\} \\
& \operatorname{star}_{Q}(C)=\{\alpha \in Q ; C \cup\{\alpha\} \in \Delta(Q)\},
\end{aligned}
$$

which should be considered as subposets of $Q$. Note that $\operatorname{link}_{\Delta(Q)}(C)=$ $\Delta\left(\operatorname{link}_{Q}(C)\right)$ and $\operatorname{star}_{\Delta(Q)}(C)=\Delta\left(\operatorname{star}_{Q}(C)\right)$. 
(2.2) Rank selection theorem. Probably one of the most important results in a theory of Cohen-Macaulay posets is the "rank selection theorem", which first appeared in Baclawski [2, Theorem 5.4] and Munkres [23, Theorem 6.4] with purely topological proofs. On the other hand, starting from the definition [28, P. 63] of Cohen-Macaulay rings by the Hilbert functions, Stanley [29, Theorem 4.3] also gave an elementary ringtheoretical proof to this theorem.

Let $Q$ be a pure poset of rank $d$. Suppose that $S$ is any subset of $[d]:=\{1,2, \cdots, d\}$. Define the rank-selected subposet $Q_{S}$ with respect to $S$ to be

$$
Q_{S}=\{\alpha \in Q ; r(\alpha) \in S\} .
$$

Note that $\operatorname{rank}\left(Q_{S}\right)=\#(S)$.

The rank selection theorem states that if $Q$ is Cohen-Macaulay then so is $Q_{S}$ for any $S \subset[d]$.

We shall prove this theorem by using $\left(^{*}\right)$ of (1.2). We may assume $S=[d]-\{i\}, i \in[d]$. Let $\left\{\alpha_{1}, \alpha_{2}, \cdots, \alpha_{n}\right\}$ be the set of all rank $i$ elements of $Q$. Then the exact sequence $\left(^{*}\right)$ asserts

$$
0 \longrightarrow \bigoplus_{i=1}^{n} k\left[\operatorname{star}_{Q}\left(\left\{\alpha_{i}\right\}\right)\right] \longrightarrow k[Q] \longrightarrow k\left[Q_{S}\right] \longrightarrow 0 .
$$

Hence $Q_{S}$ is Cohen-Macaulay by Corollary (1.3) a).

Moreover, with the same argument as in Example A of (1.4), we see that if $Q$ is Buchsbaum then so is $Q_{S}$ for all $S \subset[d]$, since $k\left[\operatorname{star}_{Q}\left(\left\{\alpha_{i}\right\}\right)\right]$ is Cohen-Macaulay by Lemma B of (1.3). This Buchsbaum analogue of the rank selection theorem was proved, in topological sence, in Baclawski [2, Theorem 6.5].

(2.3) Shellable complexes. A pure complex $\Delta$ of dimension $d$ is called shellable if its all facets (maximal faces) can be listed $F_{1}, F_{2}, \cdots, F_{s}$ in such a way that

$$
\left(\bigcup_{j=1}^{i-1} \bar{F}_{j}\right) \cap \bar{F}_{i}
$$

is pure of dimension $d-1$ for all $i>1$, where $\bar{F}_{i}=\left\{\sigma \in \Delta ; \sigma \subset F_{i}\right\}$.

If we define

$$
\sigma=\left\{v \in F_{s} ; F_{s}-\{v\} \in\left(\bigcup_{j=1}^{s-1} \bar{F}_{j}\right) \cap \bar{F}_{s}\right\},
$$

then 


$$
\begin{aligned}
& \operatorname{star}_{\Delta}(\sigma)=\bar{F}_{s}, \\
& \Delta-\{\tau \in \Delta ; \tau \supset \sigma\}=\bigcup_{j=1}^{s-1} \bar{F}_{j} .
\end{aligned}
$$

In fact, if $\tau \in \operatorname{star}_{\Delta}(\sigma)$ and $\tau \notin \bar{F}_{s}$ then $\sigma \cup \tau \in \bar{F}_{j}$ for some $j<s$, hence $\sigma \in \bar{F}_{j}$, thus $\sigma \in\left(\bigcup_{j=1}^{s-1} \bar{F}_{j}\right) \cap \bar{F}_{s}$, in other words, $\sigma \subset F_{s}-\{v\}$ for some $v \in \sigma$, a contradiction. On the other hand, $\{\tau \in \Delta ; \tau \supset \sigma\}$ coincides with $\bar{F}_{s}-\left(\bigcup_{j=1}^{s-1} \bar{F}_{j}\right) \cap \bar{F}_{s}$, thus $\Delta-\{\tau \in \Delta ; \tau \supset \sigma\}=\bigcup_{j=1}^{s-1} \bar{F}_{j}$.

Hence, thanks to $(*)$ of $(1.2)$, we have

$$
0 \longrightarrow k\left[\bar{F}_{s}\right] \longrightarrow k[\Delta] \longrightarrow k\left[\bigcup_{j=1}^{s-1} \bar{F}_{j}\right] \longrightarrow 0,
$$

so, by induction on $s$, we see that a shellable complex is Cohen-Macaulay by Corollary (1.3) b).

The notion of shellability originated in the study of polytopes. A fundamental result of Bruggesser-Main [9] concerning the shellability of the boundary complexes of simplicial polytopes is essential in McMullen [22] and Hochster [19]. The Cohen-Macaulayness of shellable complexes is essentially due to Folkman [11] in topological sense and Hochster [19] in ring-theoretical sense. See also, Björner [6], [7], Garsia [12] and KindKleinschmidt [21].

(2.4) G-complexes. Let $\Delta$ be a simplicial complex on the vertex set $V$. For $W \subset V$, define

$$
\Delta_{W}=\{\sigma \in \Delta ; \sigma \subset W\},
$$

which is a simplicial complex on $W$.

We call $\Delta$ a G-complex (or "matroid") if $\Delta_{W}$ is pure for all $W \subset V$. Refer to Stanley [27, § 7] for further information.

Note that if $\Delta$ is a $G$-complex and $\sigma \in \Delta$ then $\operatorname{link}_{\Delta}(\sigma)$ is, hence $\operatorname{star}_{\Delta}(\sigma)$ is also, a $G$-complex. To see why $\operatorname{link}_{\Lambda}(\sigma)$ is a $G$-complex, let $W$ be a subset of the vertex set of $\operatorname{link}_{4}(\sigma)$, and $\tau_{1}, \tau_{2}$ facets of $\left(\operatorname{link}_{4}(\sigma)\right)_{W}$. Then $\tau_{1} \cup \sigma, \tau_{2} \cup \sigma$ are facets of $\Delta_{W \cup \sigma}$, hence $\sharp\left(\tau_{1} \cup \sigma\right)=\#\left(\tau_{2} \cup \sigma\right)$, thus $\sharp\left(\tau_{1}\right)=\#\left(\tau_{2}\right)$.

Now, using $(*)$ and $(* *)$ of Section 1 , we shall give a direct proof, without the requirement of some combinatorial properties of $G$-complexes, to the Cohen-Macaulayness of $G$-complexes. Let $\Delta$ be a $G$-complex of dimension $d$ on the vertex set $V$. We shall prove $\Delta$ is Cohen-Macaulay by induction on $\sharp(\Delta)$. Let $v_{1}, v_{2} \in V\left(v_{1} \neq v_{2}\right)$ and $V_{i}=V-\left\{v_{i}\right\}, \Delta_{i}=\Delta_{V_{i}}$ $(i=1,2)$. We may assume $\operatorname{dim} \Delta_{i}=d$, otherwise $A=\operatorname{star}_{\Delta}\left(\left\{v_{i}\right\}\right)(i=1,2)$. 
Since $\Delta_{1} \cap \Delta_{2}=\Delta_{V-\left\{v_{1}, v_{2}\right\}}, \quad \Delta_{1} \cap \Delta_{2}$ is a $G$-complex and $\operatorname{dim}\left(\Delta_{1} \cap \Delta_{2}\right) \geq d-1$. By assumption of induction, $\Delta_{1} \cap \Delta_{2}$ is Cohen-Macaulay, hence, thanks to Corollary (1.5), $\Delta_{1} \cup \Delta_{2}$ is Cohen-Macaulay. If $\sigma=\left\{v_{1}, v_{2}\right\} \notin \Delta$, then $\Delta=$ $\Delta_{1} \cup \Delta_{2}$ and we have done. On the other hand, if $\sigma \in \Delta$ then

$$
\Delta-\{\tau \in \Delta ; \tau \supset \sigma\}=\Delta_{1} \cup \Delta_{2},
$$

hence we have the exact sequence

$$
0 \longrightarrow k\left[\operatorname{star}_{\Delta}(\sigma)\right] \longrightarrow k[\Delta] \longrightarrow k\left[\Delta_{1} \cup \Delta_{2}\right] \longrightarrow 0
$$

by $(*)$ of (1.2). Since $\operatorname{star}_{4}(\sigma)$ is a $G$-complex, $\operatorname{star}_{\Delta}(\sigma)$ is Cohen-Macaulay by assumption of induction. Hence $\Delta$ is also Cohen-Macaulay by Corollary (1.3) b).

If $\Delta$ is a $G$-complex, then the Stanley-Reisner ring $k[\Delta]$ is a "level ring" defined in Stanley [27, §3]. The concept of level rings intermediates between Cohen-Macaulay and Gorenstein which will be of interest from viewpoints of both commutative algebra and combinatorics. See [3], [16] and [35] for some results on level rings.

\section{$\S 3$. Comparability graphs and order complexes of partially ordered sets}

In order to apply Corollary (1.3) and Corollary (1.5) concerning with the Cohen-Macaulayness of simplicial complexes to finite partially ordered sets, in this section, we shall define explicitly an "intersection", a "union" and the "glueing" of a family of finite partially ordered sets.

(3.1) First, recall some definitions and terminology from graph theory.

A graph $G$ is a finite set $V \neq \varnothing$ together with a (possibly empty) set $E$ of two-element subsets of distinct elements of $V$. Each element of $V$ is referred as a vertex and $V$ itself as the vertex set of $G$ and the members of the edge set $E$ are called edges. In general, we represent the vertex set and edge set of a graph $G$ by $V(G)$ and $E(G)$, respectively. Note that, by our definition, all graphs considered in this section have no loops and multiple edges.

The edge $e=\{u, v\}$ is said to be join the vertices $u$ and $v$. If $e=\{u, v\}$ is an edge of a graph $G$, then $u$ and $v$ are adjacent vertices, while $u$ and $e$ are incident, as are $v$ and $e$. Furthermore, if $e_{1}$ and $e_{2}$ are distinct edges of $G$ incident with a common vertex, then $e_{1}$ and $e_{2}$ are adjacent edges. 
A graph $H$ is called a subgraph of a graph $G$ if $V(H) \subset V(G)$ and $E(H) \subset E(G)$. If $H$ is a subgraph of $G$, then we write $H \subset G$. Also, a graph $G$ is called complete if $\{u, v\} \in E(G)$ for all $u, v \in V(G), u \neq v$,

Let $V$ be a finite set which is a union (not necessarily a disjoint union) $\bigcup_{i=1}^{n} V_{i}$ of non-empty subsets $V_{i}$ of $V$ and $G_{i}$ graphs on the vertex sets $V_{i}(1 \leq i \leq n)$. Then we define the union $\bigcup_{i=1}^{n} G_{i}$ (resp. the intersection $\cap_{i=1}^{n} G_{i}$ ) to be a graph on the vertex set $V$ (resp. $\cap_{i=1}^{n} V_{i}$ ) whose edge set is $\bigcup_{i=1}^{n} E\left(G_{i}\right)$ (resp. $\bigcap_{i=1}^{n} E\left(G_{i}\right)$ ).

(3.2) A graph $G$ is said to be a comparability graph if there exists a partial order on $V(G)$ such that, for $u, v \in V(G), u \neq v,\{u, v\} \in E(G)$ if and only if $u$ and $v$ are comparable with respect to the partial order on $V(G)$.

By a cycle of a graph $G$ is meant here any finite sequence of vertices $v_{1}, v_{2}, \cdots, v_{n}$ of $G$ such that $\left\{v_{i}, v_{i+1}\right\}, 1 \leq i<n$, and $\left\{v_{n}, v_{1}\right\}$ are in $E(G)$, and for no vertices $\alpha$ and $\beta$ and integer $i, j<n, i \neq j, \alpha=v_{i}=v_{j}, \beta=v_{i+1}$ $=v_{j+1}$ or $\alpha=v_{i}=v_{n}, \beta=v_{i+1}=v_{1}$. A cycle is odd or even depending on whether $n$ is odd or even. By a triangular chord of a cycle $v_{1}, v_{2}, \cdots, v_{n}$ of $G$ is meant any one of the edges of the form $\left\{v_{i}, v_{i+2}\right\}, 1 \leq i \leq n-2$, or $\left\{v_{n-1}, v_{1}\right\}$ or $\left\{v_{n}, v_{2}\right\}$.

Lemma (Gilmore-Hoffmann [13]). A graph $G$ is a comparability graph if and only if each odd cycle has at least one triangular chord.

ExAMPLE. The graph $G$ of Figure 2 is not a comparability graph since an odd cycle $p, z, b, c, y$ has no triangular chord.

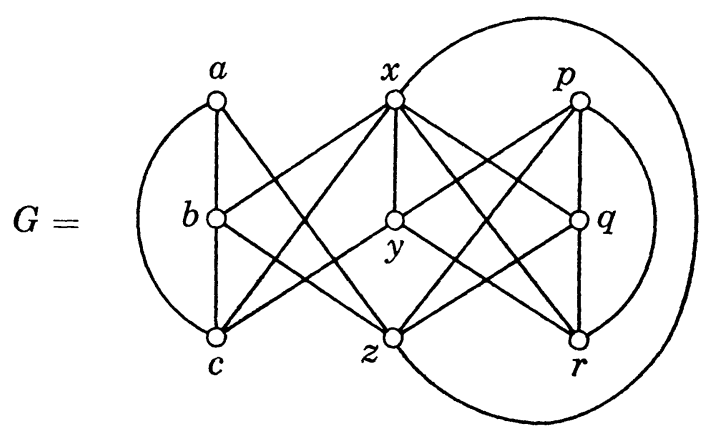

Figure 2

(3.3) Let $\Delta$ be a simplicial complex on the vertex set $V$. We will associate $\Delta$ with a graph $G(\Delta)$, called a skelton, whose vertex set is $V$ and edge set is the set of all faces of $\Delta$ with $\#(\sigma)=2$. 
For which simplicial complexes $\Delta$, does there exist a poset $Q$ such that $A=\Delta(Q)$ ? The answer was obtained in Stanley [29], namely,

Lemma (Stanley). A simplicial complex $\Delta$ is of the form $\Delta=\Delta(Q)$ for some poset $Q$ if and only if $A$ satisfies the following:

(i) the ideal $I_{\Delta}$ is generated by quadratic monomials, and

(ii) the skelton $G(\Delta)$ is a comparability graph.

In fact, the "only if" part is obvious. To see the "if" part, let the skelton $G(\Delta)$ be a comparability graph, say $G(\Delta)=G(\Delta(Q))$ for some poset Q. Then it is easy to see that $\Delta \subset \Delta(Q)$. To show $\Delta \supset \Delta(Q)$, let $\sigma \in \Delta(Q)$, namely $\sigma$ is a chain of $Q$. Then every two-element subset $\{x, y\}$ of $\sigma$ is an edge of $G(\Delta(Q))=G(\Delta)$, hence $\{x, y\} \in \Delta$. This $\sigma \in \Delta$ since $I_{\Delta}$ is generated by quadratic monomials.

(3.4) Let $V$ be a finite set which is a union $\bigcup_{i=1}^{n} V_{i}$ of non-empty subsets $V_{i}$ of $V$ and $Q_{i}$ posets whose underlying sets are $V_{i}(1 \leq i \leq n)$. Then a poset $Q$ whose underlying set is $\bigcap_{i=1}^{n} V_{i}$ is called an intersection of a family $\left\{Q_{i}\right\}_{1 \leq i \leq n}$, if $\Delta(Q)=\bigcap_{i=1}^{n} \Delta\left(Q_{i}\right)$. Of course, an intersection is not necessarily unique even though it exists.

Since the ideal $I_{\cap_{i=1}^{n} s\left(Q_{i}\right)}$ of $A=k[v ; v \in V]$ is generated by quadratic monomials, there exists an intersection of $\left\{Q_{i}\right\}_{1 \leq i \leq n}$ if and only if

$$
G\left(\bigcap_{i=1}^{n} \Delta\left(Q_{i}\right)\right)=\bigcap_{i=1}^{n} G\left(\Delta\left(Q_{i}\right)\right)
$$

is a comparability graph.

Note that the intersection of comparability graphs is not necessarily a comparability graph. For example,

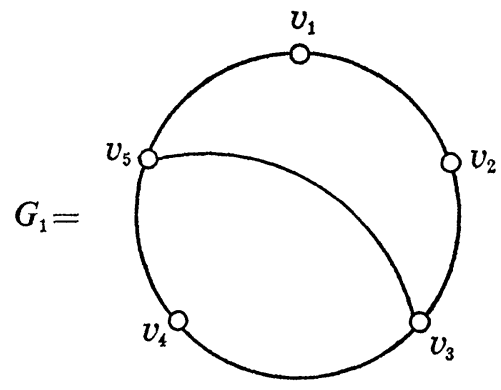

Figure 3

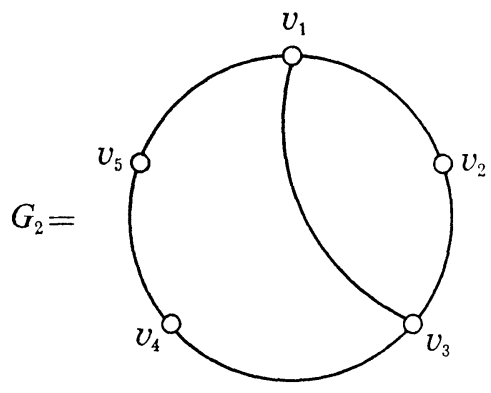

Figure 4

are comparability graphs, however, $G_{1} \cap G_{2}$ is not a comparability graph.

So, it is natural to find a sufficient condition for the existence of an 
intersection of $\left\{Q_{i}\right\}_{1 \leq i \leq n}$.

Lemma. Suppose that, for $\alpha, \beta \in \bigcap_{i=1}^{n} V_{i}$, there exist no $i$ and $j, i \neq j$, such that $\alpha<\beta$ in $Q_{i}$ while $\alpha>\beta$ in $Q_{j}$. Then there exists an intersection of $\left\{Q_{i}\right\}_{1 \leq i \leq n}$.

In fact, for $\alpha, \beta \in \bigcap_{i=1}^{n} V_{i}$, define $\alpha \leq \beta$ if $\alpha \leq \beta$ in all $Q_{i}$, and we obtain a poset $Q$ whose underlying set is $\bigcap_{i=1}^{n} V_{i}$ satisfying $\Delta(Q)=\bigcap_{i=1}^{n} \Delta\left(Q_{i}\right)$.

Of course, the condition of the above lemma is not a necessary condition.

(3.5) Now, as an analogue of a union of simplicial complexes, we will define a "union" and "glueing" of a family of partially ordered sets.

Definition. Let $V$ be a finite set which is a union $\bigcup_{i=1}^{n} V_{i}$ of nonempty subsets $V_{i}$ of $V$. Also, let $Q_{i}$ be posets whose underlying sets are $V_{i}(1 \leq i \leq n)$.

a) A poset $Q$ whose underlying set is $V$ is called a union of a family $\left\{Q_{i}\right\}_{1 \leq i \leq n}$ if $\Delta(Q)=\bigcup_{i=1}^{n} \Delta\left(Q_{i}\right)$.

b) A poset $Q$ whose underlying set is $V$ is called a glueing of a family $\left\{Q_{i}\right\}_{1 \leq i \leq n}$ if each $Q_{i}$ is a subposet of $Q$ and $\Delta(Q)=\bigcup_{i=1}^{n} \Delta\left(Q_{i}\right)$.

When does there exist a union or a glueing of a family of partially ordered sets?

Proposition. Work in the same notation as in the above definition.

a) There exists a union of $\left\{Q_{i}\right\}_{1 \leq i \leq n}$ if and only if (i) the graph $G\left(\bigcup_{i=1}^{n} \Delta\left(Q_{i}\right)\right)=\bigcup_{i=1}^{n} G\left(\Delta\left(Q_{i}\right)\right)$ is a comparability graph and (ii) every complete subgraph of $\bigcup_{i=1}^{n} G\left(\Delta\left(Q_{i}\right)\right)$ is a subgraph of $G\left(\Delta\left(Q_{i}\right)\right)$ for some $i$.

b) There exists a glueing of $\left\{Q_{i}\right\}_{1 \leq i \leq n}$ if and only if (i) for all $i \neq j$ and $\alpha, \beta \in V_{i} \cap V_{j}, \alpha \leq \beta$ in $Q_{i}$ if and only if $\alpha \leq \beta$ in $Q_{j}$ and (ii) if $\alpha_{1}, \alpha_{2} \in V_{i_{1}}$, $\alpha_{2}, \alpha_{3} \in V_{i_{2}}, \cdots, \alpha_{s}, \alpha_{s+1} \in V_{i_{s}}$ and $\alpha_{1} \leq \alpha_{2}$ in $Q_{i_{1}}, \alpha_{2} \leq \alpha_{3}$ in $Q_{i_{2}}, \cdots, \alpha_{s} \leq \alpha_{s+1}$ in $Q_{i_{s}}$, then $\left\{\alpha_{1}, \alpha_{2}, \cdots, \alpha_{s+1}\right\} \subset V_{t}$ for some $t$.

Proof. a) Let $I=I_{\cup_{i=1}^{n} \Delta\left(Q_{i}\right)}$. The problem is whether $I$ is generated by quadratic monomials or not.

First, assume that $I$ is not generated by quadratic monomials. Let $\sigma=\left\{v_{1}, v_{2}, \cdots, v_{p}\right\}(p \geq 3)$ be a subset of $V$ such that $\sigma \notin \bigcup_{i=1}^{n} \Delta\left(Q_{i}\right)$ and $\sigma-\left\{v_{j}\right\} \in \bigcup_{i=1}^{n} \Delta\left(Q_{i}\right)$ for all $j$. Then, there exists a complete subgraph $G^{\prime}$ of $\bigcup_{i=1}^{n} G\left(\Delta\left(Q_{i}\right)\right)$ whose vertex set is $\sigma$. If $G^{\prime}$ is contained in $G\left(\Delta\left(Q_{i}\right)\right.$ ) for some $i$, then $\sigma$ is a chain of $Q_{i}$, hence $\sigma \in J\left(Q_{i}\right) \subset \bigcup_{i=1}^{n} \Delta\left(Q_{i}\right)$, a contradiction. 
Conversely, assume that $I$ is generated by quadratic monomials and that $G^{\prime}$ is a complete subgraph contained in $\bigcup_{i=1}^{n} G\left(\Delta\left(Q_{i}\right)\right)$ whose vertex set is $\sigma=\left\{v_{1}, v_{2}, \cdots, v_{p}\right\}(p \geq 3)$. Then, $v_{i} v_{j} \notin I$ for all $i, j$, hence $v_{1} v_{2} \cdots v_{p} \notin I$. So, $\sigma \subset \Delta\left(Q_{i}\right)$ for some $i$. Thus $G^{\prime}$ is a subgraph of $G\left(\Delta\left(Q_{i}\right)\right)$.

b) First, we shall prove "if" part. Suppose that the condition (i) and (ii) are satisfied. Then, we can define a partial order $\leq_{V}$ on $V$ as follows. Let $v_{1}, v_{2} \in V$. Define $v_{1} \leq_{V} v_{2}$ if and only if $v_{1}, v_{2} \in V_{i}$ for some $i$ and $v_{1} \leq v_{2}$ in $Q_{i}$. By (i), this definition does not depend on the choice of $V_{i}$ containing $v_{1}$ and $v_{2}$. Also, let $v_{1}, v_{2}, v_{3} \in V$ and $v_{1} \leq_{V} v_{2}, v_{2} \leq_{V} v_{3}$. By the definition of $\leq_{V}$ we have $v_{1}, v_{2} \in V_{i}, v_{2}, v_{3} \in V_{j}$ for some $i, j$ and $v_{1} \leq v_{2}$ in $Q_{i}, v_{2} \leq v_{3}$ in $Q_{j}$. Then, $v_{1}, v_{2}, v_{3} \in V_{t}$ for some $t$ by (ii), and $v_{1} \leq v_{2}, v_{2} \leq v_{3}$ in $Q_{t}$ by (i). Hence $v_{1} \leq v_{3}$ in $Q_{t}$, this $v_{1} \leq_{V} v_{3}$. So, $\leq_{V}$ is a partial order on $V$. Let $Q$ be a poset with this partial order $\leq_{V}$ whose underlying set is $V$. Then we see immediately that $\Delta(Q)=\bigcup_{i=1}^{n} \Delta\left(Q_{i}\right)$ by (ii).

Secondly, we shall prove "only if" part. Suppose that a family $\left\{Q_{i}\right\}_{1 \leq i \leq n}$ has a glueing $Q$. Since each $Q_{i}$ is a subposet of $Q$, for $\alpha, \beta \in V_{i}$, $\alpha \leq \beta$ in $Q_{i}$ if and only if $\alpha \leq \beta$ in $Q$, hence (i) holds. Also, $\alpha_{1}, \alpha_{2} \in V_{i_{1}}$, $\alpha_{2}, \alpha_{3} \in V_{i_{2}}, \cdots, \alpha_{s}, \alpha_{s+1} \in V_{i_{s}}$ and $\alpha_{1} \leq \alpha_{2}$ in $Q_{i_{1}}, \alpha_{2} \leq \alpha_{3}$ in $Q_{i_{2}}, \cdots, \alpha_{s} \leq \alpha_{s+1}$ in $Q_{i_{s}}$, then $\alpha_{1} \leq \alpha_{2} \leq \cdots \leq \alpha_{s+1}$ in $Q$, hence $\left\{\alpha_{1}, \alpha_{2}, \cdots, \alpha_{s+1}\right\} \in \Delta(Q)=$ $\bigcup_{i=1}^{n} \Delta\left(Q_{i}\right)$. So, $\left\{\alpha_{1}, \alpha_{2}, \cdots, \alpha_{s+1}\right\} \in \Delta\left(Q_{i}\right)$ for some $i$.

Q.E.D.

ExAmple A. Let $V=\left\{v_{i}\right\}_{1 \leq i \leq 8}$ and $V_{1}=\left\{v_{i}\right\}_{1 \leq i \leq 6}, V_{2}=\left\{v_{i}\right\}_{4 \leq i \leq 8}$. Also, let

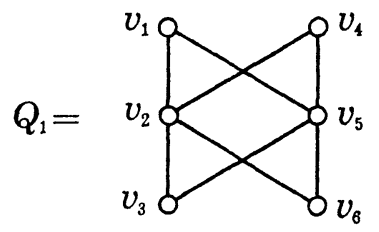

Figure 5

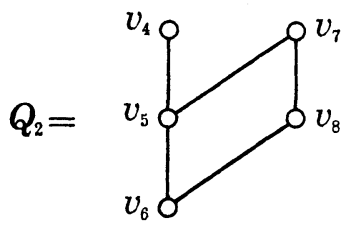

Figure 6

Then $G=G\left(\Delta\left(Q_{1}\right)\right) \cup G\left(\Delta\left(Q_{2}\right)\right)$ looks like the graph of Figure 7, which is a comparability graph. In fact, the partial order on $V$ induced by the following poset $Q$ of Figure 8 produces the above graph $G$.

So, thanks to the above proposition, we see that there exists a union of $Q_{1}$ and $Q_{2}$, however, there exists no glueing of $Q_{1}$ and $Q_{2}$.

Note that a union of a family of posets is not necessarily unique 


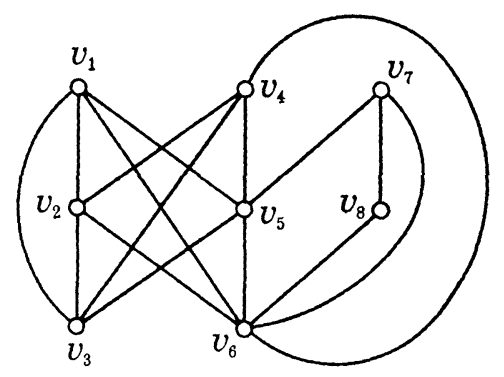

Figure 7

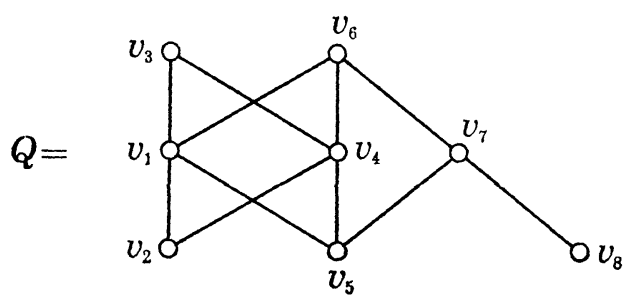

Figure 8

even though it exists, while a glueing of a family of posets is unique if it exists. Hence, from now on, we call the glueing of a family of posets.

Corollary. Let $V$ be a finite set which is a union $V_{1} \cup V_{2}$ of nonempty subsets $V_{i}$ of $V$ and $Q_{i}$ posets whose underlying sets are $V_{i}(i=1,2)$. Suppose that (i) for $\alpha, \beta \in V_{1} \cap V_{2}, \alpha \leq \beta$ in $Q_{1}$ if and only if $\alpha \leq \beta$ in $Q_{2}$ and (ii) $I_{i}:=V_{i}-\left(V_{1} \cap V_{2}\right)$ are poset ideals of $Q_{i}(i=1,2)$. Then, there exists the glueing of $Q_{1}$ and $Q_{2}$.

This corollary is treated in $[32, \S 4]$. So, our concept of the glueing of a family of posets is a generalization of that in [32].

Example B. Let $Q$ be a poset and $I, J$ poset ideals of $Q$. Then, the subposet $Q-(I \cap J)$ of $Q$ may be considered the glueing of $Q-I$ and $Q-J$. In particular, if $I \cap J=\varnothing$ then $Q$ itself is the glueing of $Q-I$ and $Q-J$.

\section{$\S 4$. Criteria of Cohen-Macaulay partially ordered sets}

The purpose of this final section is to state criteria of Cohen-Macaulay posets and to show some examples.

(3.1) To begin with, we consider 
Example. Let $Q$ be a pure poset of rank $d, i \in[d]$, and $\left\{\alpha_{1}, \alpha_{2}, \cdots\right.$, $\left.\alpha_{n}, \beta_{1}, \beta_{2}, \cdots, \beta_{m}\right\}$ the set of rank $i$ elements of $Q(n, m \geq 1)$.

Thanks to Proposition (3.5) b), two families $\left\{\operatorname{star}_{Q}\left(\left\{\alpha_{i}\right\}\right)\right\}_{1 \leq i \leq n}$ and $\left\{\operatorname{star}_{Q}\left(\left\{\beta_{j}\right\}\right)\right\}_{1 \leq j \leq m}$ have the glueings $Q_{[\alpha]}$ and $Q_{[\beta]}$, respectively. Also, $Q$ is the glueing of the family $\left\{\operatorname{star}_{Q}\left(\left\{\alpha_{i}\right\}\right), \operatorname{star}_{Q}\left(\left\{\beta_{j}\right\}\right)\right\}_{\substack{1 \leq i \leq n \\ 1 \leq j \leq m}}$.

However, $Q_{[\alpha]}$ and $Q_{[\beta]}$ are not necessarily subposets of $Q$, hence $Q$ is not necessarily the glueing of $Q_{[\alpha]}$ and $Q_{[\beta]}$. For example, if

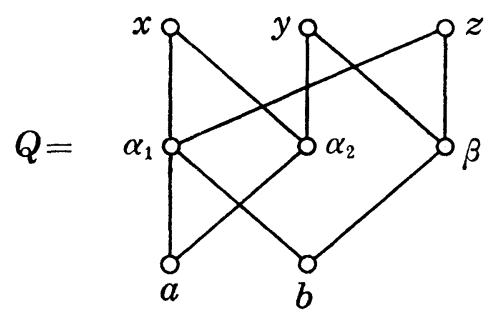

Figure 9

then

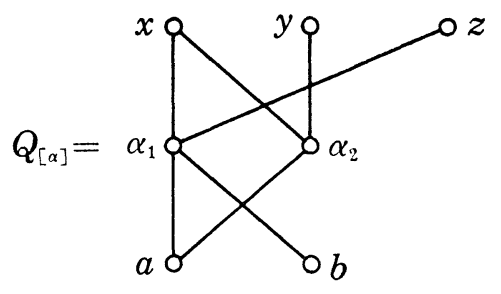

Figure 10

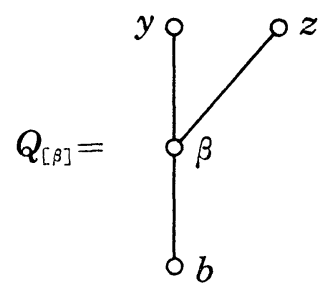

Figure 11

in this case $y$ and $b$ are comparable in $Q_{[\beta]}$ while incomparable in $Q_{[\alpha]}$, hence there exists no glueing of $Q_{[\alpha]}$ and $Q_{[\beta]}$. Of course, in general, $Q$ is a union of $Q_{[\alpha]}$ and $Q_{[\beta]}$.

Nevertheless, if $i=1$ or $d$ then $Q_{[\alpha]}$ and $Q_{[\beta]}$ are subposets of $Q$ and $Q$ is the glueing of $Q_{[\alpha]}$ and $Q_{[\beta]}$.

Note that, for each $i \in[d]$, there exists an intersection $Q^{\wedge}$ of $Q_{[\alpha]}$ and $Q_{[\beta]}$ by Lemma (3.4). For example,

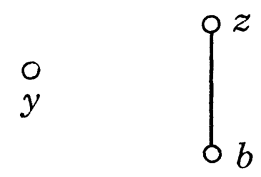

Figure 12 
is an intersection of $Q_{[\alpha]}$ of Figure 10 and $Q_{[\beta]}$ of Figure 11. In general, an intersection $Q^{\wedge}$ of $Q_{[\alpha]}$ and $Q_{[\beta]}$ is not necessarily a subposet of $Q$, however, we can take $Q^{\wedge}$ as a subposet of $Q$ if $i=1$ or $d$.

Before starting our next work, we had better introduce one notation. Let $P$ and $Q$ be posets whose underlying sets are $V_{P}$ and $V_{Q}$, respectively. We define a new poset $P \oplus Q$, called the sum, whose underlying set is the disjoint union of $V_{P}$ and $V_{Q}$ and the partial order of $P \oplus Q$ is defined by $x \leq y$ if (i) $x \leq y$ in $P$, or (ii) $x \leq y$ in $Q$, or (iii) $x \in P$ and $y \in Q$. For example,

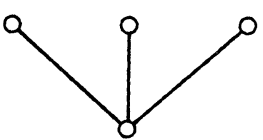

Figure 13

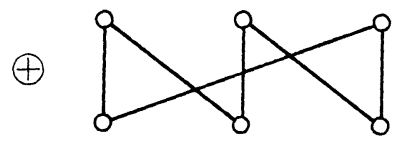

Figure 14

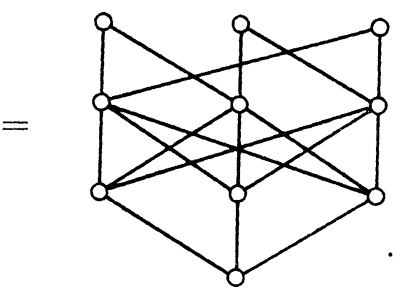

Figure 15

If $P$ and $Q$ are Cohen-Macaulay over a field $k$, then so is $P \oplus Q$ since $k[P \oplus Q]=k[P] \otimes_{k} k[Q]$.

(4.2) Now, we will state our main

THEOREM. Let $k$ be a field and $Q$ a pure poset of rank $d$.

a) (Extension lemma A) Suppose that two elements $x, y \in Q$ satisfy $x<y$ and $r(y)-r(x)=2$, and that $Q$ is Cohen-Macaulay. Then, the new poset $Q$ with $\alpha, \alpha \notin Q$, whose partial order preserves that of $Q$ and, in addition, $x^{\prime}<\alpha<y^{\prime}$ for all $x^{\prime} \leq x, y^{\prime} \geq y$, is also Cohen-Macaulay.

b) (Extension lemma B) Let $x, y$ be two elements of $Q$ satisfying $x \not y$ and $r(y)-r(x)=1$. Suppose that any element $\xi<x$ (resp. $\eta>y$ ) is comparable with $y$ (resp. $x$ ), and that $Q$ is Cohen-Macaulay. Then, the new poset $Q$ with an additional comparable relation $x<y$ is also CohenMacaulay.

c) (Union critertion) Let $i \in[d]$ and $\left\{\alpha_{1}, \alpha_{2}, \cdots, \alpha_{n}, \beta_{1}, \beta_{2}, \cdots, \beta_{m}\right\}$ the set of rank $i$ elements of $Q(n, m \geq 1)$. Also, let $Q_{[\alpha]}\left(\right.$ resp. $\left.Q_{[\beta]}\right)$ be the glueing of the family $\left\{\operatorname{star}_{Q}\left(\left\{\alpha_{i}\right\}\right)\right\}_{1 \leq i \leq n}\left(\operatorname{resp} .\left\{\operatorname{star}_{Q}\left(\left\{\beta_{j}\right\}\right)\right\}_{1 \leq j \leq m}\right)$, and $Q^{\wedge}$ an intersection of $Q_{[\alpha]}$ and $Q_{[\beta]}$. Suppose that both $Q_{[\alpha]}$ and $Q_{[\beta]}$ are CohenMacaulay over $k$. Then, $Q$ is Cohen-Macaulay over $k$ if and only if $\operatorname{depth} k\left[Q^{\wedge}\right]=d-1$. 
d) (Glueing criterion) Let $\left\{\alpha_{1}, \alpha_{2}, \cdots, \alpha_{n}, \beta_{1}, \beta_{2}, \cdots, \beta_{m}\right\}$ be the set of minimal (resp. maximal) elements of $Q(n, m \geq 1)$, and

$$
\begin{gathered}
Q_{[\alpha]}=\left\{\xi \in Q ; \xi \geq \alpha_{i} \text { for some } i\right\} \\
Q_{[\beta]}=\left\{\eta \in Q ; \eta \geq \beta_{j} \text { for some } j\right\} \\
\left(\text { resp. } \begin{array}{l}
Q_{[\alpha]}=\left\{\xi \in Q ; \xi \leq \alpha_{i} \text { for some } i\right\} \\
Q_{[\beta]}=\left\{\eta \in Q ; \eta \leq \beta_{j} \text { for some } j\right\}
\end{array}\right),
\end{gathered}
$$

which will be naturally considered as subposets of $Q$. On the other hand, let $Q^{\wedge}$ be the subposet of $Q$ consisting of all elements $x$ of $Q$ satisfying $x \geq \alpha_{i}$ and $x \geq \beta_{j}$ (resp. $x \leq \alpha_{i}$ and $x \leq \beta_{j}$ ) for some $i$ and $j$. Suppose that $Q_{[\alpha]}$ and $Q_{[\beta]}$ are Cohen-Macaulay over $k$. Then, $Q$ is Cohen-Macaulay over $k$ if and only if depth $k\left[Q^{\wedge}\right]=d-1$.

Proof. In case a) (resp. b)) we denote the new poset by $Q^{\prime}$. Since $x<y$ (resp. $\xi<y$ for all $\xi<x$ and $\eta>x$ for all $\eta>y$ ) in $Q$, we have

$$
\begin{gathered}
\Delta\left(Q^{\prime}\right)-\left\{\tau \in \Delta\left(Q^{\prime}\right) ; \tau \supset\{\alpha\}\right\}=\Delta(Q) \\
\text { (resp. } \left.\Delta\left(Q^{\prime}\right)-\left\{\tau \in \Delta\left(Q^{\prime}\right) ; \tau \supset\{x, y\}\right\}=\Delta(Q)\right),
\end{gathered}
$$

which is Cohen-Macaulay. Also,

$$
\begin{gathered}
\operatorname{star}_{Q^{\prime}}(\{\alpha\})=\{\xi \in Q ; \xi \leq x\} \oplus\{\alpha\} \oplus\{\eta \in Q ; \eta \geq y\} \\
\text { (resp. } \left.\operatorname{star}_{Q^{\prime}}(\{x<y\})=\{\xi \in Q ; \xi \leq x\} \oplus\{\eta \in Q ; \eta \geq y\}\right)
\end{gathered}
$$

is Cohen-Macaulay of rank $d$. Hence, thanks to Corollary (1.3) b), $Q^{\prime}$ is Cohen-Macaulay.

On the other hand, in case c) (resp. d)), by Example (4.1), the poset $Q$ is a union (resp. the glueing) of $Q_{[\alpha]}$ and $Q_{[\beta]}$, hence the result c) (resp. d)) is an immediate consequence of Corollary (1.5).

Q.E.D.

We have some remarks about the above results. First in case a) (resp. b)) the new poset $Q^{\prime}$ is the glueing (resp. a union) of $\operatorname{star}_{Q^{\prime}}(\{\alpha\})$ (resp. $\left.\operatorname{star}_{Q^{\prime}}(\{x<y\})\right)$ and $Q$, secondly the results a) and d) are special cases of c), and thirdly in case a) if $r(y)=2$ (resp. $r(x)=d-1$ ) then we may consider $Q \cup\{-\infty\}, x=-\infty$ (resp. $Q \cup\{\infty\}, y=\infty)$, where $-\infty$ (resp. $\infty$ ) is a least (resp. greatest) element of $Q \cup\{-\infty\}$ (resp. $Q \cup\{\infty\}$ ) which is not contained in $Q$.

Example A. In cases a) and b) the conditions concerning with $x$ and $y$ are indispensable. In fact, 


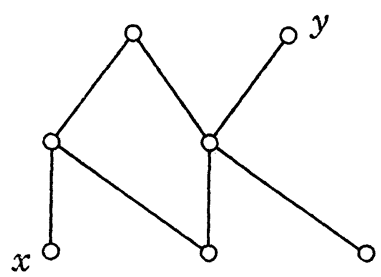

Figure 16

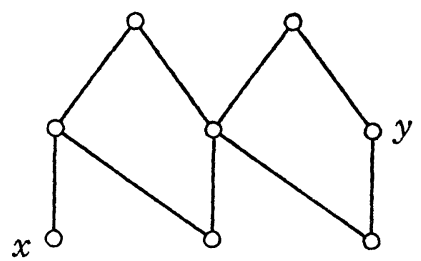

Figure 17

are Cohen-Macaulay, however,

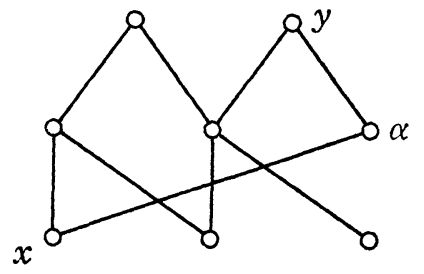

Figure 18

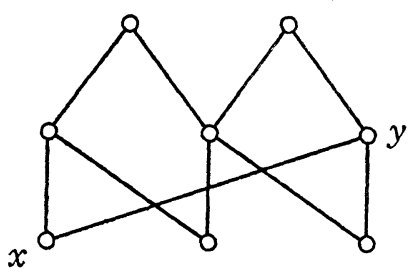

Figure 19

are not Cohen-Macaulay.

Example B. By using the union criterion c) and the glueing criterion d), we see that the following posets are not Cohen-Macaulay.

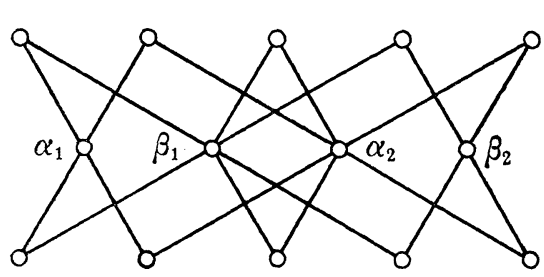

Figure 20

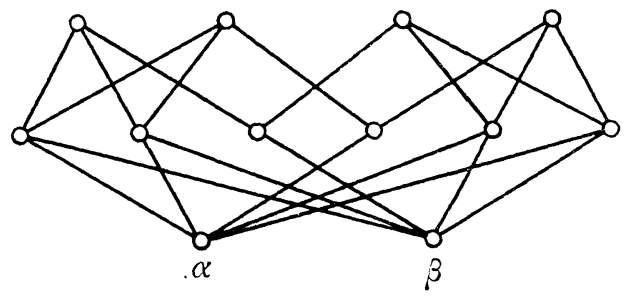

Figure 21

(4.3) Since our results of (4.2) are most fundamental in this paper, we had better consider an elementary combinatorial proof without using $(*)$ or $(* *)$ of Section 1.

In [12], Garsia obtained a purely combinatorial and linear algebraic characterization of Cohen-Macaulay posets. Recall some basic results from [12].

Let $k$ be a field, $Q$ a pure poset of rank $d$ and

$$
\theta_{i}=\sum_{r(x)=i} x
$$


the rank $i$ form in the Stanley-Reisner ring $k[Q](1 \leq i \leq d)$.

If $\mathfrak{c}=\left\{q_{1}, q_{2}, \cdots, q_{n}\right\} \subset Q$ then we denote by $\delta(\mathfrak{c})$ the square-free monomial $q_{1} q_{2} \cdots q_{n}$ of $k[Q]$. Note that $\delta(c)=0$ unless $c$ is a chain.

Let $\mathscr{C}(Q)$ be the set of all chains of $Q$ and $\mathscr{M}(Q)$ the set of all maximal chains of $Q$. For $\mathfrak{c} \in \mathscr{C}(Q)$, define

$$
L(\mathfrak{c})=\delta(\mathfrak{c}) \prod_{i \notin r(\mathfrak{c})} \theta_{i}
$$

where $r(\mathfrak{c})$ is the rank set of $c$, i.e.,

$$
r(c)=\{r(x) ; x \in c\} .
$$

Note that

$$
L(\mathfrak{c})=\sum_{\mathfrak{c} \subset \mathfrak{m} \in \mathscr{M}(Q)} \delta(\mathfrak{m}),
$$

in particular, $L(\mathfrak{m})=\delta(\mathfrak{m})$ for $\mathfrak{m} \in \mathscr{M}(Q)$.

Lemma (Garsia). In the same notation as above, $Q$ is Cohen-Macaulay over $k$ if and only if there exists a collection $\mathscr{B}(Q)$ of chains of $Q$ which satisfies

(i) $\sharp\{\mathfrak{c} \in \mathscr{C}(Q) ; r(\mathfrak{c})=S\}=\sharp\{\mathfrak{b} \in \mathscr{B}(Q) ; r(\mathfrak{b}) \subset S\}$ for all $S \subset[d]$ and

(ii) in $k[Q], L(\mathfrak{m})$ can be expressed as a linear combination $\sum_{\mathfrak{b} \in \mathscr{B}(Q)} w_{b} L(\mathfrak{b})$ $\left(w_{6} \in k\right)$ for all $\mathfrak{m} \in \mathscr{M}(Q)$.

Now, based on Garsia's characterization, we shall give an another proof to b) of Theorem (4.2). Presumably, similar proof would be given to c).

We will work in the situation of b) of Theorem (4.2). Let $T=\{r(x)$. $r(y)\}$. Since both

$$
P=\{\xi \in Q ; \xi<x\} \oplus\{\eta \in Q ; \eta>y\}
$$

and $Q$ are Cohen-Macaulay, we can choose $\mathscr{B}_{1}=\mathscr{B}(P)$ and $\mathscr{B}_{2}=\mathscr{B}(Q)$ which satisfy the conditions (i) and (ii) in the above lemma.

Let

$$
\mathscr{B}=\left\{\mathfrak{b} \cup\{x, y\} ; \mathfrak{b} \in \mathscr{B}_{1}\right\} \cup \mathscr{B}_{2} .
$$

We shall prove that this collection $\mathscr{B}$ of chains of $Q^{\prime}$ satisfies Garsia's conditions (i) and (ii) in the above lemma. In the following, the rank set of a chain $c$ of $P$ is meant the rank set as a chain of $Q$.

First, if $S \subset[d]$ and $S \not \supset T$ then 


$$
\begin{aligned}
& \sharp\left\{\mathfrak{c} \in \mathscr{C}\left(Q^{\prime}\right) ; r(\mathfrak{c})=S\right\} \\
= & \sharp\{\mathfrak{c} \in \mathscr{C}(Q) ; r(\mathfrak{c})=S\} \\
= & \sharp\left\{\mathfrak{G} \in \mathscr{B}_{2} ; r(\mathfrak{b}) \subset S\right\} \\
= & \sharp\{\mathfrak{G} \in \mathscr{B} ; r(\mathfrak{b}) \subset S\} .
\end{aligned}
$$

On the other hand, if $S \subset[d]$ and $S \supset T$ then

$$
\begin{aligned}
& \sharp\left\{\mathfrak{c} \in \mathscr{C}\left(Q^{\prime}\right) ; r(\mathfrak{c})=S\right\} \\
= & \sharp\{\mathfrak{c} \in \mathscr{C}(Q) ; r(\mathfrak{c})=S\}+\sharp\{\mathfrak{c} \in \mathscr{C}(P) ; r(\mathfrak{c})=S-T\} \\
= & \sharp\left\{\mathfrak{b} \in \mathscr{B}_{2} ; r(\mathfrak{b}) \subset S\right\}+\#\left\{\mathfrak{b} \in \mathscr{B}_{1} ; r(\mathfrak{b}) \subset S-T\right\} \\
= & \sharp\{\mathfrak{b} \in \mathscr{B} ; r(\mathfrak{b}) \subset S\} .
\end{aligned}
$$

Secondly, let $\mathfrak{m} \in \mathscr{M}\left(Q^{\prime}\right)$. If $\mathfrak{m} \supset\{x, y\}$ then

$$
\delta(\mathfrak{m}-\{x, y\}) \equiv \sum_{\mathfrak{b} \in \mathscr{P}_{1}} w_{\mathfrak{b}} \delta(\mathfrak{G}) \prod_{i \notin r(\mathfrak{b} \cup\{x, y\})} \theta_{i}
$$

in $k[Q]$ modulo the ideal generated by all $z \in Q-P$, hence

$$
\delta(\mathfrak{m}-\{x, y\})-\sum_{\mathfrak{b} \in \mathscr{W}_{1}} w_{\mathfrak{b}} \delta(\mathfrak{b}) \prod_{i \notin r(b \cup\{x, y\})} \theta_{i}=\sum_{\substack{\mathfrak{c} \in \mathscr{G}\left(\mathbb{Q}^{\prime}\right) \\ r \in \mathcal{P} \\ r(\mathfrak{c})=[d]-T}} w_{\mathfrak{r}} \delta(\mathfrak{c}) .
$$

Thus, multiplying by $x y$ on both sides, we have

$$
\delta(\mathfrak{m})=\sum_{\mathfrak{b} \in \mathscr{Q}_{1}} w_{\mathfrak{b}} \delta(\mathfrak{G} \cup\{x, y\}) \prod_{i \notin r(\mathfrak{b} \cup\{x, y\})} \theta_{i}
$$

since $\delta(\mathfrak{c}) x y=0$ if $\mathfrak{c} \not \subset P$, in other words,

$$
L(\mathfrak{m})=\sum_{\mathfrak{b} \in \mathscr{\mathscr { T }}-\mathscr{G}_{2}} w_{\mathfrak{b}} L(\mathfrak{b})
$$

as desired. On the other hand, if $m \not \supset\{x, y\}$ then

$$
L(\mathfrak{m}) \equiv \sum_{\mathfrak{b} \in \mathscr{q}_{2}} w_{\mathfrak{b}} L(\mathfrak{b})
$$

in $k[Q]$ modulo the ideal $(x y)$, hence

$$
L(\mathfrak{m})-\sum_{\mathfrak{b} \in \mathscr{g}_{2}} w_{\mathfrak{b}} L(\mathfrak{b})=\sum_{\substack{\mathfrak{m}^{\prime} \in \mathcal{M}\left(Q^{\prime}\right) \\ \mathfrak{m}^{\prime} \leq\{x, y\}}} w_{\mathfrak{n}^{\prime}} L\left(\mathfrak{m}^{\prime}\right),
$$

and the right-hand side is expressed as $\sum_{\mathfrak{b}^{\prime} \in \mathscr{A}-\mathscr{x}_{2}} w_{\mathfrak{b}^{\prime}} L\left(\mathfrak{b}^{\prime}\right)$.

Q.E.D.

(4.4) Work in the same notation as in Example (4.1).

Let $\mathscr{P}$ be a certain property for pure posets. Suppose that this property $\mathscr{P}$ satisfies the following: (i) if $Q$ is a pure poset with a unique minimal element $\alpha$ and $Q$ has this property $\mathscr{P}$, then the subposet $Q-\{\alpha\}$ 
(= $\left.\operatorname{link}_{Q}(\{\alpha\})\right)$ also has this property $\mathscr{P}$, (ii) if $Q$ is a pure poset of rank $d$ having this property $\mathscr{P}$ and $Q$ has at least two minimal elements, then for $i=1$ and some $n, m \geq 1, Q_{[\alpha]}, Q_{[\beta]}$ have $\mathscr{P}$ and $Q^{\wedge}$ is pure poset of rank $d-1$ having $\mathscr{P}$, and (iii) any chain has this property $\mathscr{P}$.

Then, by an obvious induction and d) of Theorem (4.2), we see that this property $\mathscr{P}$ implies the Cohen-Macaulay property.

ExAmple. An element $y$ of a poset $Q$ is a cover of an element $x \in Q$ if $x<y$ and no element of $Q$ is properly between $x$ and $y$.

A poset $Q$ is called wonderful if the following condition holds in the poset $Q \cup\{-\infty, \infty\}$ obtained by adjoining least and greatest elements to $Q$ : If $y_{1}, y_{2}<z$ are covers of an element $x$, then there exists an element $y \leq z$ which is a cover of both $y_{1}$ and $y_{2}$.

Now, [10, Lemma 8.2] shows that every wonderful poset is pure and that $\mathscr{P}=$ "wonderful" satisfies the above conditions (i), (ii) and (iii). Hence every wonderful poset is Cohen-Macaulay. Of course, our argument is an axiomatization of the method contained in [10, P. 42].

(4.5) We shall consider some concrete examples.

Example A. The following posets of Figure 22-24 are all CohenMacaulay. Indeed, it is a routine work to check that $Q_{i}-\left\{x_{i}\right\}$ are wonderful. Hence $Q_{i}$ are Cohen-Macaulay thanks to a) and b) of Theorem (4.2).

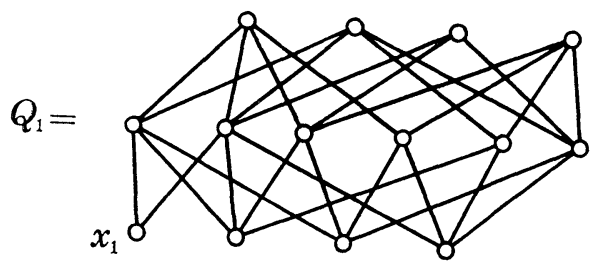

Figure 22

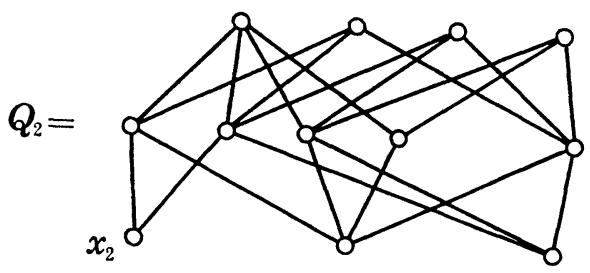

Figure 23 


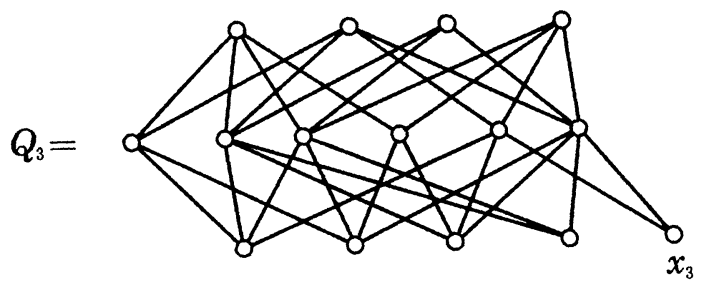

Figure 24

EXAmple B. We shall show that the poset

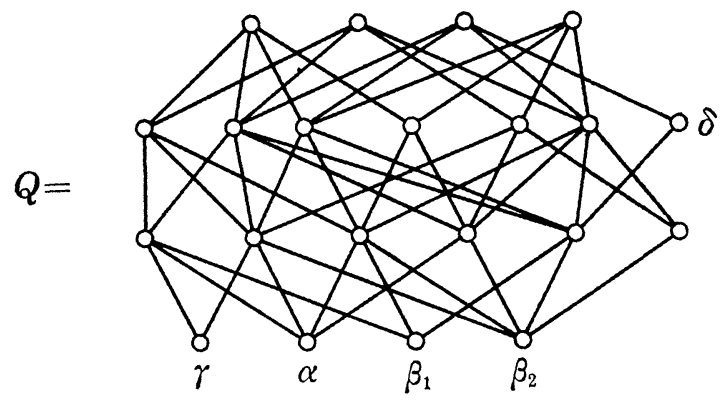

Figure 25

is Cohen-Macaulay. Let $Q^{\prime}=Q-\{\gamma, \delta\}$. Then, $\operatorname{link}_{Q^{\prime}}(\{\alpha\}), \operatorname{link}_{Q^{\prime}}\left(\left\{\beta_{1}\right\}\right)$ and $\operatorname{link}_{Q^{\prime}}\left(\left\{\beta_{2}\right\}\right)$ coincide with $Q_{1}, Q_{2}$ and $Q_{3}$ in the above Example A, respectively.

Since an intersection of $\operatorname{star}_{Q^{\prime}}\left(\left\{\beta_{1}\right\}\right)$ and $\operatorname{star}_{Q^{\prime}}\left(\left\{\beta_{2}\right\}\right)$ looks like

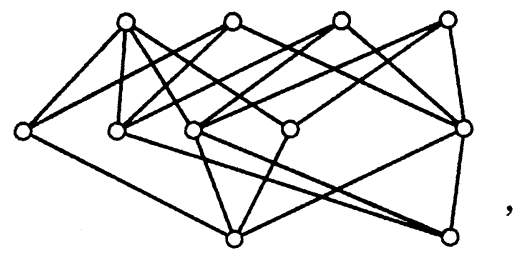

Figure 26

which is a wonderful poset of rank 3 , the glueing $Q_{[\beta]}^{\prime}$ of $\operatorname{star}_{Q^{\prime}}\left(\left\{\beta_{1}\right\}\right)$ and $\operatorname{star}_{Q^{\prime}}\left(\left\{\beta_{2}\right\}\right)$ is Cohen-Macaulay. On the other hand, the poset $Q_{1}$ of Example A, which is Cohen-Macaulay of rank 3, is an intersection of $Q_{[\beta]}^{\prime}$ and $\operatorname{star}_{Q^{\prime}}(\{\alpha\})$, hence the poset $Q^{\prime}$, which is the glueing of $Q_{[\beta]}^{\prime}$ and $\operatorname{star}_{Q^{\prime}}(\{\alpha\})$, is Cohen-Macaulay. So, thanks to a) and b) of Theorem (4.2) again, we see that $Q$ is Cohen-Macaulay.

Let $\Pi_{n}$ be the partially ordered set of partitions of an integer $n$ ordered 
by refinement, see Birkhoff [5, I, Example 10, P. 16]. According to Björner [6, Example 6.2], $\Pi_{n}$ is wonderful if and only if $n \leq 7$. On the other hand, $\Pi_{8}$ is not wonderful but is shellable, namely, the simplicial complex $\Delta\left(\Pi_{8}\right)$ is shellable. In general, it is proved in [6, Theorem 6.1] that if $Q$ is a wonderful poset then $\Delta(Q)$ is shellable. See also Björner-Wachs [8].

Here, as an application of Theorem (4.2), we shall prove the CohenMacaulayness of $\Pi_{9}$ directly.

The poset $Q^{(0)}=\Pi_{9}-\{x, y, z\}$, where $x=(\underbrace{1,1, \cdots, 1}_{9 \text {-times }}), y=(\underbrace{1, \cdots, 1}_{7 \text {-times }}, 2)$, $z=(9)$, is just the poset of Figure 1 .

First, we shall prove $Q^{(1)}=Q^{(0)}-\{(1,1,1,1,1,1,3),(1,1,1,1,1,2,2)$, $(1,1,1,1,1,4)\}$ is Cohen-Macaulay. Let $\alpha=(1,1,1,1,2,3)$ and $\beta=$ $(1,1,1,2,2,2)$ of $Q^{(1)}$. Then the poset $\operatorname{link}_{Q^{(1)}}(\{\alpha\})$ is just the poset of Figure 25 of Example $B$, hence $\operatorname{link}_{Q^{(1)}}(\{\alpha\})$ is Cohen-Macaulay, thus $\operatorname{star}_{Q^{(1)}}(\{\alpha\})$ is Cohen-Macaulay. On the other hand, by the same method

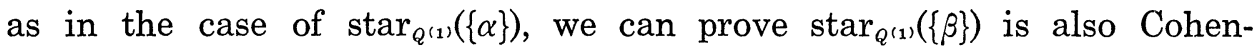
Macaulay.

Now, an intersection of $\operatorname{star}_{Q^{(1)}}(\{\alpha\})$ and $\operatorname{star}_{Q^{(1)}}(\{\beta\})$ looks like

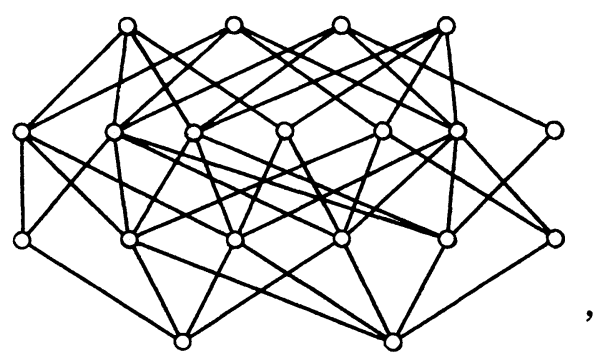

Figure 27

which can be checked to be Cohen-Macaulay by the glueing criterion d) of Theorem (4.2), hence, again thanks to d) of Theorem (4.2), $Q^{(1)}$ is Cohen-Macaulay.

Secondly, apply the extension lemmas a) and b) of Theorem (4.2) to $Q^{(1)}$ and $(1,1,1,1,1,4)$, and we see that $Q^{(2)}=Q^{(0)}-\{(1,1,1,1,1,1,3)$, $(1,1,1,1,1,2,2)\}$ is Cohen-Macaulay. Hence $Q^{(3)}=Q^{(0)}-\{(1,1,1,1,1,1,3)\}$ is also Cohen-Macaulay.

Finally, again applying a) and b) of Theorem (4.2) to $Q^{(3)}$ and $(1,1,1,1,1,1,3), Q^{(0)}$ is Cohen-Macaulay, which means $\Pi_{9}$ is CohenMacaulay.

Q.E.D. 
After this paper was submitted, the author received Björner's letter of May 8, 1986, in which the recent paper Ziegler [33] was referred. According to [33], both $\Pi_{9}$ and $\Pi_{10}$ are shellable, however, for $n \geq 19$, the posets $\Pi_{n}$ are not Cohen-Macaulay.

The author would like to thank Prof. A. Björner for his information about the partition posets $\Pi_{n}$.

(4.6) The final topic of this paper is to consider a question that what conditions of partially ordered sets ensure Buchsbaum posets to be Cohen-Macaulay.

We will propose the following

Definition. A pure poset $Q$ of rank $d$ is called an $L^{(n)}$-poset, where $n$ is a positive integer, if for each $i \in[d]$ there exist $n$ elements $\alpha_{1}^{(i)}, \alpha_{2}^{(i)}$, $\cdots, \alpha_{n}^{(i)}$ of $\operatorname{rank} i$ such that (i) $\alpha_{1}^{(1)}, \alpha_{2}^{(1)}, \cdots, \alpha_{n}^{(1)}$ are all the minimal elements of $Q$ and (ii) for each $r \in[d-1]$ and $s>1$,

$$
\left(\bigcup_{j=1}^{s-1}\left[\alpha_{j}^{(r)}, \infty\right)\right) \cap\left[\alpha_{s}^{(r)}, \infty\right)=\bigcup_{j=1}^{s}\left[\alpha_{j}^{(r+1)}, \infty\right)
$$

where $[\alpha, \infty)=\{x \in Q ; x \geq \alpha\}$ for $\alpha \in Q$.

Lemma. Let $Q$ be an $L^{(n)}$-poset. Suppose that the subposets $\left[\alpha_{j}^{(1)}, \infty\right)$ of $Q$ are Cohen-Macaulay for all $j, 1 \leq j \leq n$. Then, the subposet $\bigcup_{j=1}^{s}\left[\alpha_{j}^{(1)}, \infty\right)$ of $Q$ is Cohen-Macaulay for every $s, 1 \leq s \leq n$. In particular, $Q$ itself is Cohen-Macaulay.

Proof. We shall prove by induction on $d$. The case $d=1$ is trivial. Let $d>1$. Since $\left[\alpha_{j}^{(1)}, \infty\right)$ is Cohen-Macaulay for every $j,\left[\alpha_{j}^{(2)}, \infty\right)$ is Cohen-Macaulay for every $j$. Hence, by assumption of induction, the subposets $\bigcup_{j=1}^{s}\left[\alpha_{j}^{(2)}, \infty\right)$ of $Q$ are Cohen-Macaulay for all $s$. Now, we shall prove each subposet $\bigcup_{j=1}^{s}\left[\alpha_{j}^{(1)}, \infty\right)$ of $Q$ is Cohen-Macaulay by induction on $s$. Let $s>1$. Since $\bigcup_{j=1}^{s-1}\left[\alpha_{j}^{(1)}, \infty\right)$ and $\left[\alpha_{s}^{(1)}, \infty\right)$ are Cohen-Macaulay, thanks to the glueing criterion d) of Theorem (4.2), $\bigcup_{j=1}^{s}\left[\alpha_{j}^{(1)}, \infty\right)$ is CohenMacaulay, because the subposet

$$
\left(\bigcup_{j=1}^{s-1}\left[\alpha_{j}^{(1)}, \infty\right)\right) \cap\left[\alpha_{s}^{(1)}, \infty\right)=\bigcup_{j=1}^{s}\left[\alpha_{j}^{(2)}, \infty\right)
$$

of $Q$, which may be regarded as an intersection of $\bigcup_{j=1}^{s-1}\left[\alpha_{j}^{(1)}, \infty\right)$ and $\left[\alpha_{s}^{(1)}, \infty\right)$, is Cohen-Macaulay of rank $d-1$.

Q.E.D.

Now, combining the above lemma with Lemma B of (1.3), we obtain 
Proposition. Let $Q$ be an $L^{(n)}$-poset. Then $Q$ is Cohen-Macaulay if and only if $Q$ is Buchsbaum.

ExAmple. Let $\Pi_{n}$ be the partition poset of the integer $n \geq 4$ considered in (4.5). Also, let $\Pi_{(n)}=\Pi_{n}-\left\{x_{n}, y_{n}, z_{n}\right\}$, where

$$
x_{n}=(\underbrace{1,1, \cdots, 1}_{n \text {-times }}), \quad y=(\underbrace{1, \cdots, 1}_{(n-1) \text {-times }}, 2), \quad z_{n}=(n) .
$$

Then, $\Pi_{(n)}$ is an $L^{(2)}$-poset of rank $n-3$. In fact, it is easy to see that

$$
\begin{aligned}
& \alpha_{1}^{(i)}=(\underbrace{1,1, \cdots, 1}_{(n-(i+2)) \text {-times }}, i+2) \\
& \alpha_{2}^{(i)}=(\underbrace{1,1, \cdots, 1}_{(n-(2+3)) \text {-times }}, 2, i+1),
\end{aligned}
$$

$i \in[n-3]$, satisfy the conditions (i) and (ii) in our definition of $L^{(2)}$-posets.

\section{REFERENCES}

[1] K. Baclawski, "Homology and combinatorics of ordered sets", Thesis, Harvard University, 1976.

[ 2 ] — Cohen-Macaulay ordered sets, J. Algebra, 63 (1980), 226-258.

[ 3 ] — Cohen-Macaulay connectivity and geometric lattices, European J. Comb., 3 (1982), 293-305.

[4] K. Baclawski and A. Garsia, Combinatorial decompositions of a class of rings, Adv. in Math., 39 (1981), 155-184.

[5] G. Birkhoff, "Lattice Theory", 3rd. ed., Amer. Math. Soc. Colloq. Publ., No. 25, Amer. Math. Soc., Providence, R.I., 1967.

[6] A. Björner, Shellable and Cohen-Macaulay partially ordered sets, Trans. Amer. Math. Soc., 260 (1980), 159-183.

[7] - Some combinatorial and algebraic properties of Coxeter complexes and Tits buildings, Adv. in Math., 52 (1984), 173-212.

[ 8 ] A. Björner and M. Wachs, On lexicographically shellable posets, Trans. Amer. Math. Soc., 277 (1983), 323-341.

[9] H. Bruggesser and P. Mani, Shellable decompositions of cells and spheres, Math. Scand., 29 (1971), 197-205.

[10] C. De Concini, D. Eisenbud and C. Procesi, Hodge Algebras, Astérisque, 91 (1982).

[11] J. Folkman, The homology groups of a lattice, J. Math. Mech., 15 (1966), 631-636.

[12] A. Garsia, Combinatorial methods in the theory of Cohen-Macaulay rings, Adv. in Math., 38 (1980), 229-266.

[13] P. Gilmore and A. J. Hoffmann, A characterization of comparability graphs and interval graphs, Canad. J. Math., 16 (1967), 539-548.

[14] S. Goto and K.-i. Watanabe, On graded rings, I, J. Math. Soc. Japan, 30 (1978), 179-213.

[15] T. Hibi, Distributive lattices, affine semigroup rings and algebras with straightening laws, to appear in the proceeding of USA-JAPAN joint seminar on Commutative Algebra and Combinatorics held at Kyoto (August, 1985).

[16] — Level rings and algebras with straightening laws, to appear. 
[17] T. Hibi and K.-i. Watanabe, Study of three-dimensional algebras with straightening laws which are Gorenstein domains I, Hiroshima Math. J., 15 (1985), 27-54.

[18] _ Study of three-dimensional algebras with straightening laws which are Gorenstein domains II, Hiroshima Math. J., 15 (1985), 321-340.

[19] M. Hochster, Rings of invariants of tori, Cohen-Macaulay rings generated by monomials, and polytopes, Ann. of Math., 96 (1972), 318-337.

[20] - Cohen-Macaulay rings, combinatorics and simplicial complexes, Ring Theory II, Proc. Second Oklahoma Conf. (March, 1976), Lect. Notes in Pure and Appl. Math., No. 26, Dekker, 1977, 171-223.

[21] B. Kind and P. Kleinschmidt, Schälbara Cohen-Macaulay Komplexe und ihre Parametrisierung, Math. Z., 167 (1979), 173-179.

[22] P. McMullen, The maximal number of faces of a convex polytope, Mathematika, 17 (1970), 179-184.

[23] J. Munkres, Topological results in combinatorics, Michigan Math. J., 31 (1984), $113-128$.

[24] G. Reisner, Cohen-Macaulay quotients of polynomial rings, Adv. in Math., 21 (1976), 30-49.

[25] P. Schenzel, On the number of faces of simplicial complexes and the purity of Frobenius, Math. Z., 178 (1981), 125-142.

[26] R. Stanley, The upper bound conjecture and Cohen-Macaulay rings, Studies in Appl. Math., 54 (1975), 135-142.

[27] - Cohen-Macaulay complexes, Higher Combinatorics (M. Aigner, editor), NATO Advanced Study Institute Series, Reidel, Dordrecht and Boston, 1977, 51-62.

[28] — - Hilbert functions of graded algebras, Adv. in Math., 28 (1978), 57-83.

[29] —-, Balanced Cohen-Macaulay complexes, Trans. Amer. Math. Soc., 249 (1979), 139-157.

[30] — "Combinatorics and Commutative Algebra", Progress in Math., Vol. 41, Birkhäuser, 1983.

[31] J. Stückrad and W. Vogel, Toward a theory of Buchsbaum singularities, Amer. J. Math., 100 (1973), 727-746.

[32] K.-i. Watanabe, Study of algebras with straightening laws of dimension 2, Algebraic and Topological Theories-to the memory of Dr. Takehiko Miyata (M. Nagata et al., eds.), Kinokuniya, Tokyo, 1985, 622-639.

[33] G. Ziegler, On the poset of partitions of an integer, J. Combin. Theory, Series A, 42 (1986), 215-222.

[34] T. Hibi, Plane graphs and Cohen-Macaulay posets, submitted.

[35] — Regular and semi-regular points of Cohen-Macaulay partially ordered sets, submitted.

[36] — Canonical ideals of Cohen-Macaulay partially ordered sets, to appear.

Department of Mathematics

Faculty of Science

Nagoya University

Chikusa-ku, Nagoya 464

Japan 